\title{
Scintillator Surface Detector simulations for AugerPrime
}

\author{
David Schmidt ${ }^{1, *}$ for the Pierre Auger Collaboration ${ }^{2, * *, * * *}$ \\ ${ }^{1}$ Karlsruhe Institute of Technology (KIT) \\ ${ }^{2}$ Observatorio Pierre Auger, Av. San Martín Norte 304, 5613 Malargüe, Argentina
}

\begin{abstract}
Knowledge of the mass composition of ultra-high-energy cosmic rays is understood to be a salient component in answering the open questions in the field. The AugerPrime upgrade of the Pierre Auger Observatory aims to enhance its surface detector with the hardware necessary to reconstruct primary mass for individual events. This involves placing a scintillation-based detector with an active area of $3.8 \mathrm{~m}^{2}$ on top of each existing water-Cherenkov detector in its surface detector array. Here, we present the methods for simulating this Scintillator Surface Detector. These simulations have and will continue to aid in the interpretation of measurements with AugerPrime as well as the development and improvement of event reconstruction algorithms including primary mass.
\end{abstract}

\section{Introduction}

The Pierre Auger Observatory (Auger) [1], with its unprecedented exposure of over $60000 \mathrm{~km}^{2} \mathrm{sr} \mathrm{yr}$ acquired during more than decade of data collection, has led to progress in the field of Ultra-High-Energy Cosmic Rays (UHECR) physics in a number of ways. To name a couple, the suppression in the energy spectrum above approximately $40 \mathrm{EeV}$ has been confirmed to high precision [2, 3], and a large-scale dipole anisotropy above $8 \mathrm{EeV}$ has been clearly observed [4]. With these advances, however, Auger has also uncovered additional and unexpected complexity. Perhaps most notable is the trend towards heavier composition at the highest energies, which reopens questions regarding the origins of the flux suppression. Additionally, the long standing mystery as to the origins of UHECRs remains unsolved, and the additional complication of mass now presents further challenges. For this reason, the observatory is embarking on its next phase by equipping its Surface Detector (SD) array with the hardware necessary to estimate the mass of UHECRs on an event-by-event basis.

This upgrade, known as AugerPrime [5, 6], capitalizes on the fact that the magnitude of the muonic component of Extensive Air Showers (EASs) produced by UHECRs scales with primary mass. AugerPrime aims to disentangle the contributions of the electromagnetic and muonic shower components in SD measurements, thereby obtaining a mass-sensitive estimator. This is being accomplished through the installation of an Scintillator Surface Detector (SSD) on top of each of the existing Water-Cherenkov Detectors (WCDs) that make up the SD array. The relatively higher sensitivity of the SSDs (WCDs) to particles

\footnotetext{
*e-mail: david.schmidt@kit.edu

**e-mail: auger_spokespersons@fnal.gov

***Authors list: http://www.auger.org/archive/authors_2018_10.html
}

of the electromagnetic (muonic) air shower components allows for this disentanglement.

A prototype array of SSDs has been operating in the field since September of 2016, and deployment of production design SSDs for the complete array is currently underway. In order to aid in understanding and interpreting measurements of the upgraded SD, as well as develop reconstruction algorithms, we have developed comprehensive SSD simulations. The methods used in these simulations are described in this proceeding and build upon [7].

\section{Scintillator Surface Detector}

The active area of an SSD is comprised of 48 polystyrene scintillator bars measuring $1600 \mathrm{~mm}$ by $50 \mathrm{~mm}$ with a thickness of $10 \mathrm{~mm}$. This makes for an active area of just over $3.8 \mathrm{~m}^{2}$, which is split between two wings of 24 bars each (see Fig. 1). $1 \mathrm{~mm}$ diameter Wave-Length Shifting (WLS) fibers of $5.8 \mathrm{~m}$ length are routed through two bean shaped holes inside each bar with a U-bend of $10 \mathrm{~cm}$ diameter at the ends furthest from the central axis of the SSD such that both fiber ends may be routed into a cookie situated between the two scintillator wings. There are approximately $1.25 \mathrm{~m}$ of fiber between the central ends of the scintillator bars and the cookie, and the lengths of the fibers are uniform for all scintillator bars through which they are routed. The bars and fibers are housed within an aluminum casing whose bottom consists of a composite panel with $22 \mathrm{~mm}$ of Extruded Polystyrene (XPS) situated between two $1 \mathrm{~mm}$ sheets of aluminum. The top of the housing consists of a $1 \mathrm{~mm}$ sheet of aluminum. The space within the SSD housing not occupied by the bars and fibers is mostly filled with Expanded Polystyrene (EPS), resulting in a volume of air inside the detector of less than $10 \mathrm{~L}$. For a detailed description of the detector materials, geometry, and construction, see [8]. 


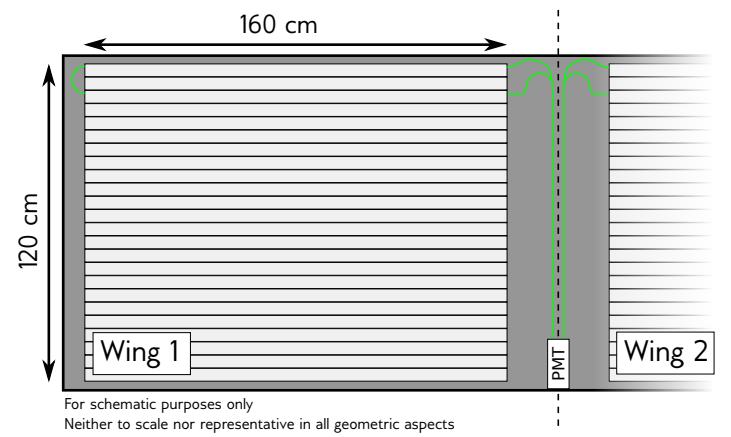

Figure 1. Top-down schematic depiction of the arrangement of bars and fibers within the SSD casing. The written dimensions describe the active surfaces of the detectors, namely the scintillator bars.

\section{Simulation Application}

The SSD simulation application is housed within Auger's official simulation and reconstruction framework, Offline [9], which was upgraded to accommodate for the demands of the large-scale detector upgrade that is AugerPrime [7]. The SSD simulations are based in the field-standard simulation software Geant4 [10] (version 4.10), but, where it is possible, make use of measurements of SSDs performed with a centimeter-precision muon telescope for enrichment and tuning. The combined use of Geant 4 and the muon telescope measurements has made highly detailed simulation of the detector possible. The simulations account for the energy-loss processes of traversing particles, the decays occurring from excited states of atoms within the scintillator bars and WLS fibers, the attenuation of photons along the fibers, the Photoelectrons (PEs) generated at the photo-cathode of the Photomultiplier Tube (PMT), the current at the base of the PMT, and the response of the AugerPrime station electronics [11]. The following sections step through the construction of the detector in Geant4 and each stage of the simulation.

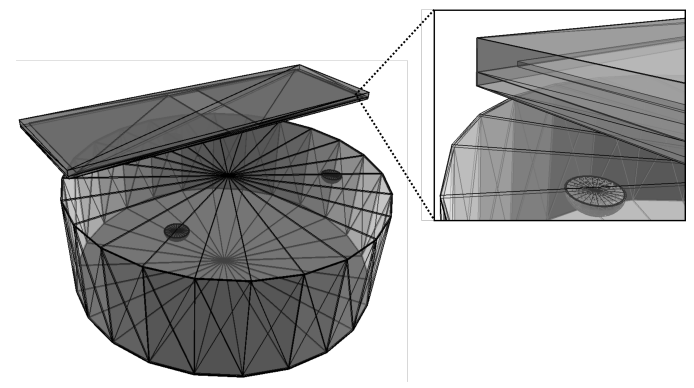

Figure 2. Visualization of both the SSD and WCD volumes defined in the Geant4-based simulation application with a close-up of one corner of the SSD

\subsection{Detector Construction}

SSDs are constructed within the same Geant 4 world volume as their partner WCDs. The volumes for both detectors may be observed in Fig. 2. Regarding the SSD, volumes for the aluminum casing, XPS, EPS, and scintillator bars themselves are defined according to the dimensions and material properties of the production SSD design, described in Sec. 2. This is important to accurately reproduce the shielding above and below the active area of the scintillator bars. Precise definition of the position of the SSD relative to the WCD is important for preserving signal correlations, as these depend on the fraction of particles that intersect both detectors.

\subsection{Photoelectrons}

For each particle that intersects or is generated within the active scintillator volumes, the energy deposited within the bars is extracted from Geant 4 and converted into a number of photoelectrons at the photo-cathode of the PMT. This conversion must take into account the attenuation of photons within the bars and along the fibers as well as the excitations and decays corresponding to scintillation and wavelength-shifting processes. The conversion takes the form of

$$
\bar{N}(\mathbf{x})=N_{\text {ref }} \frac{E_{\text {dep }}}{E_{\text {ref }}} f_{\text {att }}(\mathbf{x})
$$

where $E_{\mathrm{dep}}$ is the energy deposit in the scintillator bars, $E_{\text {ref }}$ and $N_{\text {ref }}$ are the reference energy and PE number derived from simulations, and $f_{\text {att }}(\mathbf{x})$ describes the degree of photon attenuation as a function of particle crossing position $\mathbf{x} . E_{\text {ref }}$ is the peak in the distribution of energy deposited in the SSD by an ensemble of vertical, throughgoing muons simulated for the volumes described in Sec. 3.1. $N_{\text {ref }}$ is a constant derived such that average value of Eqn. 1 when integrated over all possible values of $\mathbf{x}$ inside the scintillator bars is equal to the the average number of PEs observed in real measurements of the SSDs with the aforementioned muon telescope. For a more detailed description of how these parameters were obtained, see [7]. With this construction, the mean number of PEs for a single, through-going muon is guaranteed to match real measurements. The attenuation function $f_{\text {att }}(\mathbf{x})$ is written as

$$
f_{\mathrm{att}}(\mathbf{x})=A(\mathbf{x}) L(\mathbf{x}),
$$

where $A(\mathbf{x})$ describes the attenuation along the WLS fibers. This attenuation is of the form

$$
A(\mathbf{x})=e^{-\ell_{\mathrm{c}}(\mathbf{x}) / \lambda_{\mathrm{f}}}+e^{-\ell_{\mathrm{f}}(\mathbf{x}) / \lambda_{\mathrm{f}}},
$$

where the two exponential terms correspond to the two paths photons may travel to reach the PMT due to the Ubend routing of the fibers. $\ell_{\mathrm{c}}\left(\ell_{\mathrm{f}}\right)$ corresponds to the shorter (longer) travel path and is calculated for a given crossing position $\mathbf{x} . \lambda_{\mathrm{f}}$ is the effective attenuation length of the fiber obtained through analysis of muon telescope SSD measurements. The term $L(\mathbf{x})$ corresponds to a decreased yield at crossing positions very close the edges of the scintillator bars. Thus, for any energy deposit and particle crossing position, an expected number of PEs corresponding to each of the two paths photons may travel to reach the PMT may be calculated. A Poisson randomization is then performed on each of these expectations. For each PE coming out of these randomizations, two draws, one from each of 

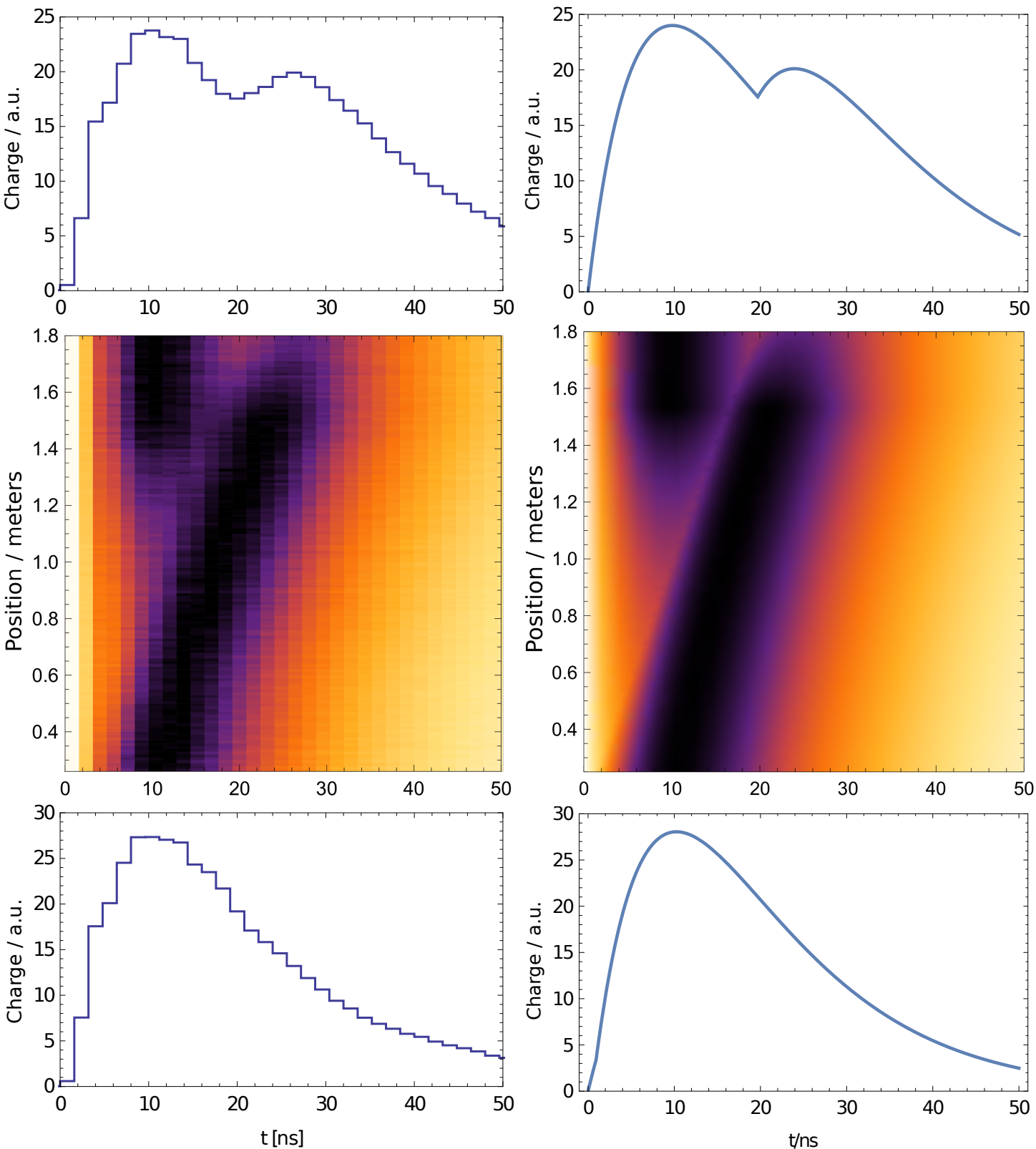

Figure 3. Depiction of the time evolution of the signal from single, through-going muons in an SSD. Muon telescope measurements are shown in the plots on the left and the parameterization of these measurements are shown in those on the right. Middle: Density plot of the signal evolution over one scintillator wing of the SSD. The bottoms of the histograms correspond to a particle crossing at the non-central edges of the scintillator bars, where photons corresponding to the two legs of the signal traverse approximately equal distance and therefore produce a unimodal distribution. The tops of the histograms correspond to the central edges of the scintillator bars, where the photons corresponding to the two legs of signal traverse significantly different distances and therefore result in a bimodal distribution. Slices in the horizontal axis have been normalized to the same maximum. Top and bottom: Signal shapes at the central and non-central ends of the scintillator bars.

two exponential distributions are performed. This model of two exponential decays, which may arbitrarily be attributed to the excitation associated with scintillation in the scintillator bars and the wave-length shifting process in the WLS fibers, was found to provide a sufficient description of the data [7]. The production time for each PE is then obtained via

$$
t_{\mathrm{PE}}=t_{\mathrm{global}}+t_{\mathrm{kin}}+t_{\mathrm{bar}}+t_{\text {fiber }}
$$

where $t_{\text {global }}$ is the global time obtained from Geant $4, t_{\text {kin }}$ is the kinematic delay of the photon as it traverses its respective path to the PMT, and $t_{\mathrm{bar}}$ and $t_{\mathrm{fiber}}$ are the times arising from the two aforementioned exponential decays. The average measured and parameterized time distributions for crossing positions along the complete active surface of one wing of the SSD, which compound Eqn. 1, Eqn. 4, and the convolution of the two exponential decays, may be observed in Fig. 3. 

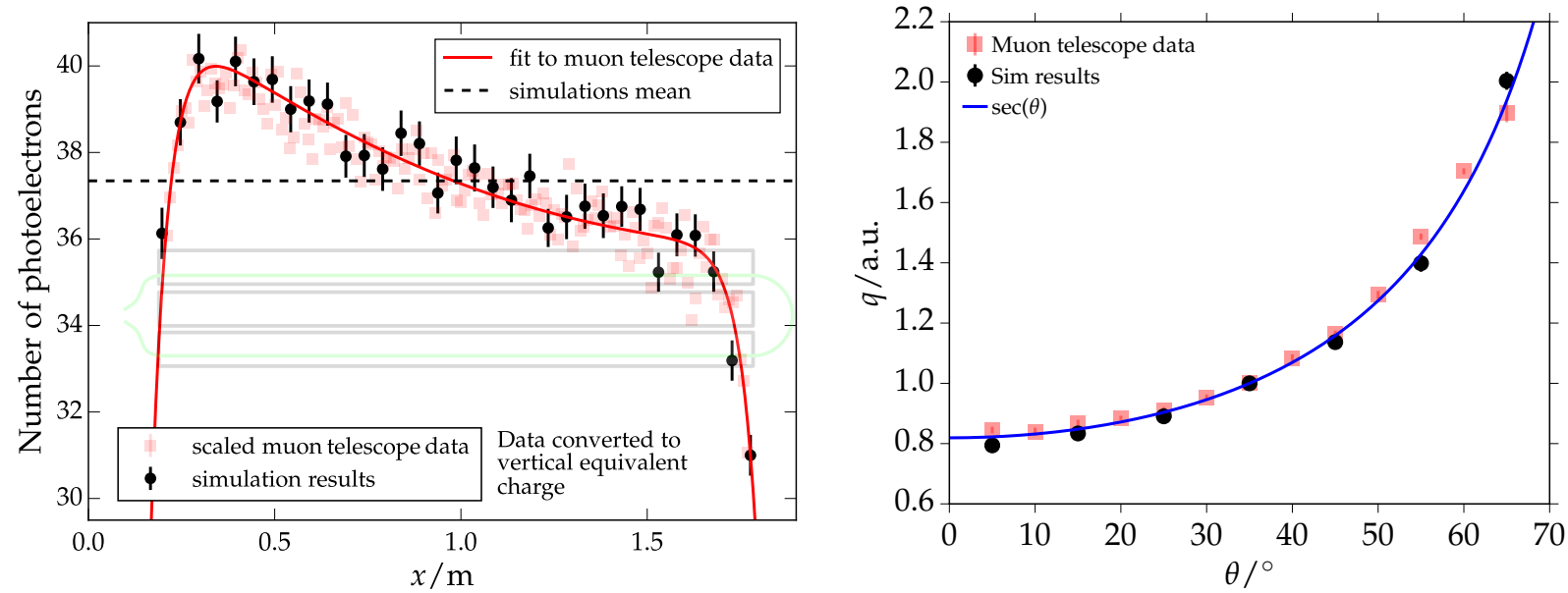

Figure 4. Left: Average charge measured for single, through-going muons as a function of the crossing position for one wing of an SSD. Measurements with the muon telescope are shown by the square, red markers, whereas simulated data is represented by the circular, black markers. Larger values on the horizontal axis correspond to crossing positions further from the central edge of the scintillator, as depicted by the semi-transparent schematic in the backdrop. Right: Average charge as a function of zenith angle. Once more, muon telescope measurements are given by the red markers, whereas simulations are shown in black. Both data sets have been scaled such that they equal one at an angle of $35^{\circ}$ to compare their evolution with tracklength $(1 / \cos \theta)$.

\subsection{PMT and electronics}

With a trace of PE production times in hand, the PMT is simulated as follows. For each PE, the average pulse shape at the PMT base corresponding to an Single Photoelectron (SPE) is scaled by a random draw from the corresponding charge distribution. Both this pulse shape and the charge distribution were measured for SPE events for the PMT in question, as described in [7]. Performing this process for each PE results in a time distribution of the electrical current at the base of the PMT, which is the input to the upgraded SD station electronics [11].

The effect of the electronics on the time trace is simulated by convolving the base current distribution with the measured electronics transfer function. This results in an analog voltage trace, which is then scaled, sampled, and digitized. The scaling is performed such that the resulting peak in the charge distribution for a simulated ensemble of through-going muons equals that which has been measured by prototype SSDs deployed in an Engineering Array (EA) [12] at the observatory site near Malargüe since 2016. The sampling and digitization are performed in accordance with the $120 \mathrm{MHz} 12$ bit design of the AugerPrime electronics.

\section{Validation}

Validation of the output of the simulations was performed on a per-particle basis by comparing the positiondependent charge and time distributions of simulated muons to those measured by the muon telescope. In Fig. 4 , the dependencies of charge on crossing position and zenith angle of the particles are compared between measurements and simulations. In both cases, the simulations provide a reasonable description of the data. A full validation closely examining the shapes of the charge and time distributions is currently underway as are additional comparisons with measurements of SSDs deployed in the EA.

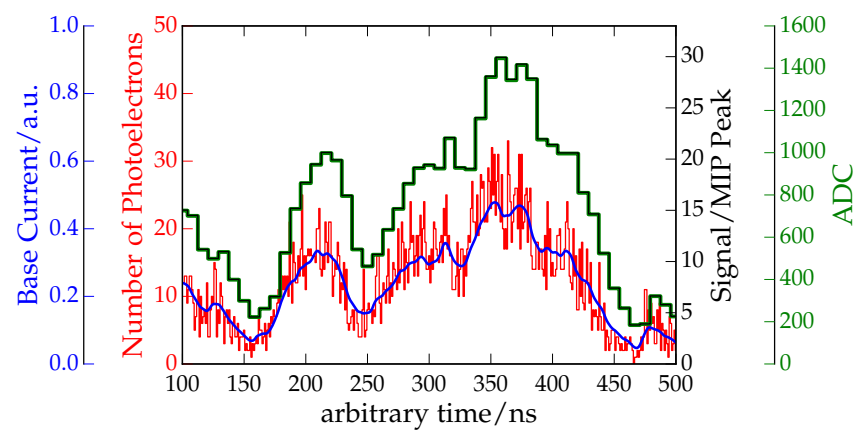

Figure 5. Simulated SSD measurement. Time traces for PE production, current at the base of the PMT, post-electronics digitized signals, and calibrated to the signal of one Vertical Minimum Ionizing Particle (VMIP) are shown.

\section{Example Application}

For a given simulated extensive air shower, the particles reaching ground near each SD station are injected into a cylindrical volume that completely encases both the SSD and WCD. Propagation of these incident particles into the shared volume and subsequent interactions and generation of new particles is then left to the Geant4 software, and signals are simulated as described in Sec. 3. A depiction of time distributions of photoelectrons, current at the base of the PMT, and digital signals after electronics processing are shown in Fig. 5 for an example station simulation. An example event, where SSD and WCD pairs sample the lateral distribution of particles at ground at various locations, based on Auger's hexagonal grid, is depicted in Fig. 6. 


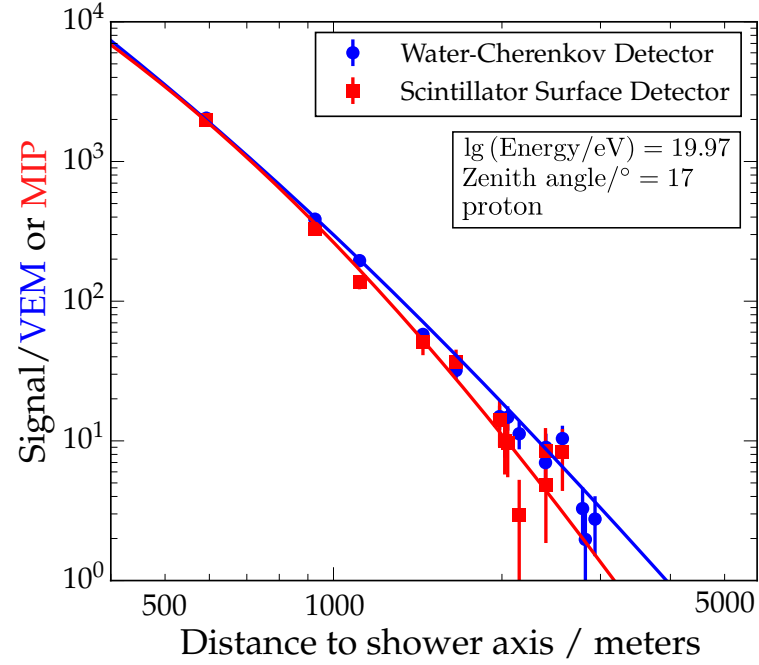

Figure 6. Simulated signals of SSDs alongside partner WCDs for various samples of the lateral distribution of the particles reaching ground in a simulated EAS.

\section{Conclusions}

Detector simulations for the SSD of the AugerPrime upgrade have been developed and the methods, making use of Geant 4 and real detector measurements, have been described here. Initial validation has shown general agreement between the detector simulations and centimeterprecision muon telescope measurements for the charge and time distributions produced by single, through-going muons. Additional, more detailed, validation is currently being performed. The combined SSD and WCD simulation application, housed in the $\overline{\mathrm{Off}}$ line detector simulation and reconstruction software framework of Auger, has already and will continue to aid in the interpretation of measurements performed with AugerPrime as well as in the development and improvement of event reconstruction algorithms which include primary mass.

\section{References}

[1] The Pierre Auger Collaboration, Nucl. Instrum. Meth. A 798, 172-213 (2015)

[2] The Pierre Auger Collaboration, Phys. Rev. Lett. 101, 061101 (2008)

[3] The Pierre Auger Collaboration, Phys. Lett. B 685, 239-246 (2010)

[4] The Pierre Auger Collaboration, Science 357, 12661270 (2017)

[5] The Pierre Auger Collaboration, arXiv:1604.03637 (2016)

[6] Daniele Martello for the Pierre Auger Collaboration, Proc. 35th Int. Cosmic Ray Conf. PoS(ICRC2017) 486, (2017)

[7] D. Schmidt for the Pierre Auger Collaboration, PoS ICRC2017, 353 (2017)

[8] R. Smida for the Pierre Auger Collaboration, PoS ICRC2017, 390 (2017)

[9] S. Argiro et al., Nucl. Instrum. Meth. A 580, 1485 1496 (2007)

[10] S. Agostinelli et al., Nucl. Instrum. Meth. A 506, 250-303 (2003)

[11] T. Suomijärvi for the Pierre Auger Collaboration, PoS ICRC2017, 450 (2017)

[12] Z. Zong for the Pierre Auger Collaboration, PoS ICRC2017, 449 (2017) 